\title{
Autoria e empacotamento de conteúdos
}

\author{
Alessandra Pereira Rodrigues ${ }^{1}$ \\ Mary Lúcia Pedroso Konrath ${ }^{2}$ \\ Liane Margarida Rockenbach Tarouco ${ }^{3}$ \\ Adelina Mezzari ${ }^{4}$
}

\begin{abstract}
This paper is the result of a research that investigates authoring tools, wich allow the construction of contents and its packaging for use and reuse in different contexts by different individuals, trying to achieve the pedagogical objectives of a unit of learning, as well as, achieve their students' educational needs. This study was conducted through the experiences of packaging Learning Objects (LOs) from some of the patterns presented, the usage of a eXe authoring tool for the production and integration of a LO into the Moodle environment. The main issue here was studying the behavior of the LOs produced by authoring tools investigated in the Moodle LMS. Our results presents that the authoring tools analized allow the encapsulation of objects in at least one of the patterns, SCORM or IMS, through export options available within their tools. The LOs available in these formats facilitate the production, reuse, tracking and managing the access of those students as well as allow the teacher to adapt to different contexts and learning situations.
\end{abstract}

Keywords: authorship, content packaging, standardization

Resumo. O presente artigo é resultado de uma pesquisa que investiga ferramentas de autoria, as quais permitem a construção de conteúdos e seu empacotamento para uso e reuso em diferentes contextos e por diferentes sujeitos, buscando atender os objetivos pedagógicos de uma unidade de aprendizagem, assim como atendendo às necessidades educacionais de seus alunos. Este estudo foi realizado através de experiências de empacotamento de Objetos de Aprendizagem (OAs) a partir de alguns dos padrões apresentados, exploração da ferramenta de autoria eXe para produção e integração de um OA no ambiente Moodle. A questão central de pesquisa buscou estudar o comportamento dos OAs produzidos pelas ferramentas de autoria investigadas frente ao LMS Moodle. Como principais resultados têm-se que as ferramentas de autoria estudadas permitem o encapsulamento de objetos em pelo menos um dos padrões, SCORM ou IMS, através de opções de exportação disponíveis nas próprias ferramentas. Os OAs disponibilizados nestes formatos facilitam a produção, reuso, rastreamento e gerenciamento quanto ao acesso dos alunos, assim como possibilitam a adaptação pelo professor a diferentes contextos e situações de aprendizagem.

Palavras-chave: autoria, empacotamento de conteúdos, padronização

\section{Introdução}

Avanços tecnológicos têm aumentado as oportunidades de comunicação e interação através da Internet. Objetos de aprendizagem (OAs) neste contexto são blocos de conteúdos com os quais professores podem organizar situações de aprendizagem, buscando atender os objetivos pedagógicos de uma unidade de aprendizagem, assim como atendendo às necessidades educacionais de seus alunos. O projeto e criação destes OAs envolvem o uso de linguagens e ferramentas de autoria e esta construção demanda elevada quantidade de tempo e recursos multimídia. Neste sentido, é importante planejar adequadamente o projeto, a construção e a gestão destes recursos como forma de torná-los acessíveis a diferentes contextos. $\mathrm{Na}$ construção de OAs têm-se cada vez mais buscado desenvolver conteúdos que sejam independentes de uma única ferramenta de autoria, ambiente virtual de aprendizagem (LMS) ou de plataforma de hardware e software, mas que atendam as especificações técnicas do Comitê de Padrões de Tecnologia de Aprendizagem. Com este objetivo, tem sido utilizadas

\footnotetext{
${ }_{1}^{1}$ Professora no Instituto Federal de Educação, Ciência e Tecnologia Sul-rio-grandense, doutoranda no Programa de Pós-graduação em Informática na Educação da UFRGS. Email: alesspr@gmail.com

${ }^{2}$ Professora de Informática no Colégio Mãe de Deus e doutoranda no Programa de Pós-graduação em Informática na Educação da UFRGS. Email: marykonrath@globo.com

${ }_{3}^{3}$ Professora CINTED-UFRGS e PGIE-UFRGS. Email: liane@penta.ufrgs.br

${ }^{4}$ Professora na Faculdade de Farmácia da UFRGS, professora na UFCSPA, pós-doutoranda no Programa de Pósgraduação em Informática na Educação da UFRGS. Email: mezzari@ufcspa.edu.br

V. $7 \mathrm{~N}^{\mathrm{o}} 3$, dezembro, 2009
} 
ferramentas de autoria para produção e encapsulamento de OAs que possam ser disponibilizados em diferentes ambientes virtuais de aprendizagem.

Esse artigo busca apresentar os estudos sobre o comportamento dos OAs, produzidos pelas ferramentas de autoria investigadas, frente ao LMS Moodle. Esta investigação procura responder as seguintes questões de pesquisa:

- Como as ferramentas estudadas permitem o encapsulamento de OAs?

- Como os objetos gerados pelas ferramentas de autoria estudadas se integram ao LMS?

- Como o LMS registra (avaliação formativa e somativa) a interação dos alunos com o objeto?

A pesquisa foi realizada através de experiências de empacotamento de OAs a partir de alguns dos padrões apresentados, exploração de ferramentas de autoria para produção de OAs e integração destes no ambiente Moodle.

O artigo está dividido em 6 seções. Nesta primeira seção está descrito o contexto, o problema a ser investigado, as questões de pesquisa, a abordagem metodológica e a organização do artigo. Na segunda seção são apresentados os principais padrões de empacotamento existentes. Na terceira são apresentadas algumas ferramentas que permitem a produção de OAs e seu empacotamento nos padrões referenciados na seção anterior. $\mathrm{Na}$ seção seguinte é apresentado um estudo de caso, na área de Micologia do curso de Medicina da UFCSPA, com produção de material educacional digital produzido através da ferramenta de autoria eXe. Na quinta seção são apresentados os ambientes que aceitam OAs empacotados segundo os padrões analisados. E por fim, são apresentadas na última seção as conclusões deste artigo seguidas das referências que embasaram esta pesquisa.

\section{Padrões de Empacotamento}

Nesta seção trataremos dos padrões mais disseminados de empacotamento de objetos de aprendizagem.

Empacotar um objeto de aprendizagem significa reunir todos os arquivos que compõe um OA num mesmo arquivo, em conformidade com um padrão e com informações a respeito dos metadados e dos arquivos que compõe o mesmo.

No sentido de buscar padrões no empacotamento, instituições como o Comitê de Padrões de Tecnologia de Aprendizagem (LTSC - Learning Technology Standards Committee), o projeto Aliança de Autoria de Aprendizagem a Distância e Redes de Distribuição na Europa (ARIADNE - Alliance of Remote Instructional Authoring and Distribution Networks for Europe) e os Sistemas de Gerenciamento Instrucional (IMS - Instructional Management Systems) foram as primeiras a desenvolver padrões técnicos para apoiar a ampla disseminação dos objetos de aprendizagem.

A ideia da padronização é alcançar a interoperabilidade entre sistemas de diferentes origens. Um ponto importante da padronização não é impor uma particular implementação, mas em vez disso a especificação comum estabelecendo oportunidades de colaboração por diversos grupos. (HARMAN e KOOHANG, 2007b)

Nas subseções seguintes serão discutidas a importância de padronizar OAs e resumidas as características de quatro padrões de empacotamento de objetos, sendo o ICOPER um padrão em desenvolvimento.

\subsection{Importância da Padronização}

A importância de padronizar os OAs se dá devido à necessidade de reutilização de conteúdos educacionais digitais, minimizando esforços na produção desses materiais e permitindo a utilização de OAs existentes na formação de um novo OA que atenda os objetivos pedagógicos da situação de aprendizagem que o professor deseja aplicar.

Além de preocupar-se com a construção de OAs padronizados, na concepção do conteúdo educacional o autor deve ter em mente as características de granularidade e independência de contexto, planejando adequadamente as sequências instrucionais que são pontos cruciais para o uso bem sucedido de objetos de aprendizagem.

A granularidade diz respeito ao grau de detalhamento/precisão contido em um $O A$, assim como o seu tamanho e possibilidade de decomposição. A definição de granularidade proposta por Koohang e Harman (2007a) para um OA útil e válido é que ele seja 


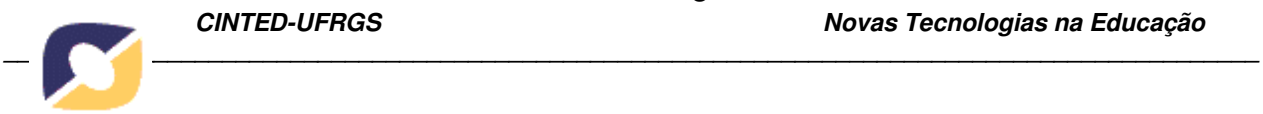

composto por uma apresentação digital (texto, vídeo, áudio, imagens, animações) que pode ser razoavelmente discernida, ou seja, que tenha uma explicação acompanhando e que responda no mínimo a uma questão dos níveis da taxonomia de Bloom (1956) de funcionamento cognitivo para reforçar a informação e explicação, uma explicação e uma estrutura global do objeto que utiliza uma ou mais teorias de aprendizagem no projeto. A independência de contexto pode ser obtida através de planejamento e desenvolvimento de pequenos OAs genéricos e que possam se adaptar a situações de aprendizagem diferentes. Quanto mais genérico for o conteúdo mais situações de aprendizagem serão contempladas.

$\mathrm{Na}$ seção a seguir, serão destacados alguns dos padrões de empacotamento existentes.

\subsection{Alguns padrões existentes}

O padrão LOM (Learning Object Metadata standard) é o pioneiro e um dos mais difundidos, considerado a base para os demais padrões de empacotamento existentes. Esse padrão tem como objetivos habilitar alunos para pesquisa e uso de OAs e habilitar agentes de computador para compor automaticamente OAs para cada aluno. O padrão LOM especifica um modelo conceitual que define a estrutura da instância de metadados para um objeto de aprendizagem. (HARMAN e KOOHANG, 2007b)

Outro padrão muito difundido é o SCORM. SCORM é a abreviatura de Sharable Content Object Reference Model que é um modelo de referência, ou seja, um conjunto de padrões e especificações modelo para a agregação, sequenciamento e execução de objetos de aprendizagem para uso online, utilizados principalmente em cursos de capacitação, treinamento e formação na modalidade de Educação a Distância. (ADVANCED DISTRIBUTED LEARNING, 2004)

O padrão SCORM permite:

- Padronização dos conteúdos;

- Reutilização, portabilidade, usabilidade e interoperabilidade dos objetos de aprendizagem e

- Flexibilização da aprendizagem.

Como uma de suas principais vantagens têm-se a questão de não estar restrito a uma única plataforma (Learning Management System), sendo possível ser utilizado por todas que forem compatíveis com este modelo.

O padrão SCORM permite o empacotamento de conteúdo, que consiste em agregar todos os objetos de aprendizagem de uma unidade de aprendizagem em um único local, definidos a partir de uma determinada sequência e contendo os metadados deste conjunto. Ao final deste processo, através da "scormização" do conteúdo, este se transforma em um arquivo único compactado denominado de pacote, o qual poderá ser importado para os ambientes virtuais de aprendizagem compatíveis, tal como o Moodle.

Um terceiro padrão analisado neste artigo é o IMS (Instructional Management Systems), um projeto da IMS Learning Global Consortium que visa sugerir padrões e modelos para a indústria de aprendizagem. Os objetos de aprendizagem foram definidos por este padrão em três aspectos pedagógicos: (1) objetivos, (2) conteúdo instrucional e (3) prática e feedback. São construídos baseados na linguagem de modelagem educacional (EML Educational Modelling Language).

Entre os modelos desta organização têm-se:

- $\quad$ IMS Content Packaging (IMS-CP)

- $\quad$ IMS Learning Design (IMS-LD)

Dentre os modelos que a IMS possui, o foco deste artigo é o IMS LD, que é um padrão aberto integrado a outras especificações, com o objetivo de modelar objetos e atividades de aprendizagem. O IMS LD dá suporte ao uso de diferentes abordagens do processo de ensinoaprendizagem, tais como: behavioristas, cognitivistas e construtivistas. Esta possibilidade acontece a partir de uma linguagem genérica e flexível, projetada para abranger diversos tipos de abordagens pedagógicas com a mesma tecnologia. O modelo descreve "Unidades de Aprendizagem", como unidades elementares que provêem eventos de aprendizagem para aprendizes, satisfazendo um ou mais objetivos de aprendizagem. 
E por último, um padrão ainda em desenvolvimento, mas com uma proposta inovadora, é o ICOPER $^{\mathbf{5}}$ que significa Interoperable COntent for PERformance, um consórcio de instituições européias, com a missão de recolher e desenvolver as melhores práticas para conteúdos interoperáveis que apóie o ensino superior baseado em competência.

O projeto ICOPER investiga as seguintes atividades desenvolvidas nas Instituições de Ensino Superior:

- Necessidades de Aprendizagem e Oportunidades de Planejamento;

- Modelagem Instrucional;

- Desenvolvimento de Conteúdo;

- Distribuição de Aprendizagem e

- Avaliação.

O consórcio investiga os padrões e especificações existentes, como:

- OpenID, Open Archives Initiative Protocol for Metadata Harvesting (OAI-PMH), Simple Publishing Interface (SPI), DOI/OpenURL;

- XML Schemas of Human Resource XML (HR-XML), Open Applications Group Integration Specification (OAGIS), IEEE Reusable Competency Definitions (IEEE RCD);

- $\quad$ IMS Learning Design (LD);

- ISO/IEC 19796-1:2005 (Information technology for learning, education and training quality management, assurance and metrics);

- $\quad$ IMS Learning Design (LD), IMS Content Packaging (CP);

- $\quad$ IMS Question \& Test Interoperability (QTI) e

- SCORM.

\section{Ferramentas que implementam os padrões estudados}

Nesta seção serão descritas algumas ferramentas de autoria de materiais educacionais digitais que permitem o empacotamento dos materiais produzidos utilizando algum padrão existente, como por exemplo, SCORM ou IMS.

\section{$3.1 \mathrm{eXe}$}

O eXe - elearning XTML editor é uma ferramenta de autoria para a produção de materiais educacionais digitais para a Web, livre e de código aberto, disponível para download nas versões dos sistemas operacionais Windows, Linux e Macintosh.

$\mathrm{O}$ eXe foi desenvolvido com o objetivo de assistir professores e acadêmicos na publicação de conteúdo para Web sem que estes necessitem de conhecimentos aprofundados nas linguagens HTML e XHTML.

Os conteúdos produzidos por esta ferramenta podem ser exportados para vários formatos, como: IMS Content Package, Common Cartridge, SCORM 1.2, pasta de conteúdo com o conjunto de arquivos no formato de páginas web ou pasta de conteúdo no formato zip, simples página web auto-contida, no formato txt e no formato de notas para iPod.

A ferramenta eXe permite a criação de Objetos de Aprendizagem ou Unidades de Aprendizagem, constituídos por iDevices, que são dispositivos instrucionais disponíveis para 0 autor escolher de acordo com a proposta pedagógica de cada atividade do OA.

Por padrão, alguns iDevices vem carregados junto a ferramenta, como: ampliador de imagem, applet java, artigo wiki, atividade, atividade lacuna, atividade leitura, estudo de caso, galeria de imagens, múltipla escolha, objetivos, pré-requisitos, questão verdadeiro ou falso, RSS, reflexão, SCORM Quiz (somativo), seleção múltipla, sítio web externo e texto livre. É possível criar novos iDevices através do editor de iDevices disponível na ferramenta. Os iDevices construídos serão templates e poderão ser utilizados em outras instalações, através das opções de exportação e importação, existentes também no referido editor. A figura 1 mostra a caixa com todos os iDevices disponíveis no eXe.

\footnotetext{
5 Mais informações através do site http://www.icoper.org/project-objectives
} 

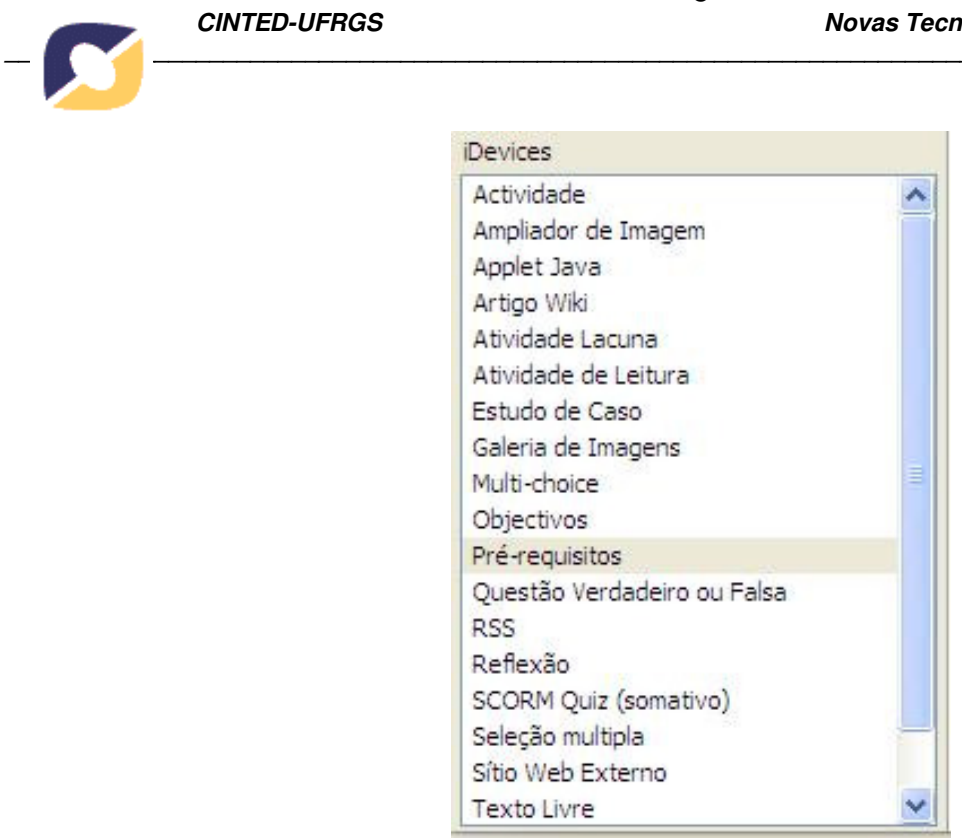

Figura 1 - iDevices disponíveis no eXe

Como foi dito anteriormente, não é necessário ter conhecimentos de linguagens de programação para construir materiais educacionais utilizando o eXe, mas caso o usuário deseje construir o material utilizando a linguagem HTML é possível, através de um botão disponível na barra de ferramentas do editor de textos do iDevice, como mostra a figura 2.

Os iDevices possuem diferenças no que se referem a sua constituição e ao seu objetivo de uso. Um iDevice é formado por caixas de texto com um editor embutido do tipo WYSIWYG, que permite a inclusão de imagens, vídeos, links, tabelas e textos, como mostra a figura 2.

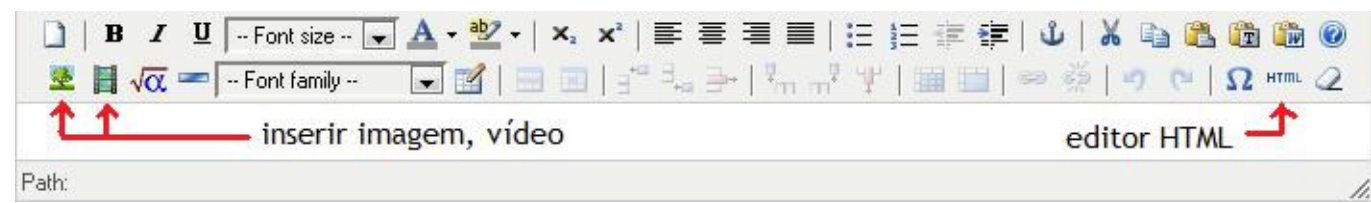

Figura 2: Editor dos iDevices do eXe

Outra diferença existente entre os iDevices é referente a avaliação e a rastreabilidade da navegação do usuário pela Unidade de Aprendizagem. Neste sentido, o único iDevice que possui as duas características é o SCORM Quiz (somativo), que permite ao próprio usuário verificar dentro da Unidade de Aprendizagem por onde já navegou e as partes que ainda faltam navegar, como será examinado no estudo de caso da seção 4.

Um Objeto de aprendizagem é definido por Wiley (2002) como "...qualquer recurso digital que pode ser reusado para apoiar a aprendizagem". Neste contexto e acrescentando que um OA pode estar disponível em várias situações de aprendizagem, considerando sua característica principal de ser um recurso reusável, definimos aqui como uma Unidade de Aprendizagem sendo um conjunto de objetos de aprendizagem integrados e com um contexto definido de acordo com objetivos pedagógicos.

Considerando que objetos de aprendizagem podem ser pequenas porções de conteúdo de uma unidade de aprendizagem, uma boa prática na utilização da ferramenta eXe é construir objetos pequenos e que sejam o mais genérico possível, para que os mesmos possam ser reutilizados em várias situações de aprendizagem diferentes. Assim, uma unidade de aprendizagem pode ser construída com vários OAs criados previamente. Para isto, a ferramenta disponibiliza a opção de incluir um pacote pré-existente do próprio eXe. Uma desvantagem em construir unidades de aprendizagem completas é que estas serão pouco reutilizadas, pois estarão focadas em uma única situação de aprendizagem. 


\subsection{ReLoad}

ReLoad significa Reusable e-Learning Object Authoring \& Delivery, um projeto focado no desenvolvimento de ferramentas baseadas nas especificações de interoperabilidade e tecnologia de aprendizagem. É gerenciada pela Universidade de Bolton.

Basicamente, a ferramenta ReLoad permite a criação e o empacotamento de objetos de aprendizagem seguindo algum padrão existente.

O projeto ReLoad ${ }^{6}$ oferece a comunidade 5 ferramentas para download, com o objetivo de construir OAs em conformidade com os padrões mais utilizados. As ferramentas, bem como os padrões que cada um deles atendem estão descritos a seguir:

- The Classic ReLoad Editor: suporta os padrões IMS Metadata, IEEE LOM, IMS Content Packaging 1.1.4, SCORM 1.2 e SCORM 2004.

- The Eclipse-based RELOAD Editor: suporta os padrões IMS MD (versions 1.1, 1.2.1 and 1.2.4), IEEE LOM, IMS CP (versions 1.1.1, 1.1.2 and 1.1.4), SCORM 1.2 e SCORM 2004 (3 $3^{\text {a }}$ edição).

- Learning Design Editor: suporta todas as especificações IMS.

- SCORM 1.2 Player: visualiza um pacote no formato SCORM.

- Learning Design Player: visualiza uma unidade de aprendizagem LD (Learnind Design)

\subsection{Moodle - Livro}

O livro é um módulo do LMS Moodle que é disponibilizado na forma de um recurso e permite criar um livro eletrônico que pode ser constituído em forma de capítulos e sub-capítulos, sendo possível a sua impressão total ou apenas de um capítulo.

Este recurso também permite gerar um pacote no formato IMS CP, possibilitando que o mesmo possa ser disponibilizado em outro curso no próprio LMS Moodle ou em outro LMS que aceite este padrão de empacotamento.

As limitações do livro referem-se ao uso colaborativo do recurso, por dois motivos: o primeiro refere-se ao recurso não prever acesso concorrente na edição de páginas do livro e o segundo refere-se ao sistema de permissões que restringe a edição apenas a professores, comprometendo desta forma atividades colaborativas por alunos com uso deste recurso. Uma das maneiras de contornar o segundo motivo é a criação de um curso onde os alunos são cadastrados com os privilégios de professores, possibilitando dessa forma que editem 0 conteúdo do livro.

\subsection{Outras ferramentas}

Hotpotatoes, CourseLab e Thesis são outras ferramentas de autoria para a produção de materiais educacionais digitais que empacotam os OAs em algum padrão.

O Hotpotatoes é uma ferramenta gratuita para uso educacional, mas não livre, que permite criar exercícios interativos de cinco tipos: múltipla escolha, resposta curta, palavrascruzadas, ordenação de sentenças e preencher lacunas. Permite ainda criar uma unidade didática com a combinação dos exercícios anteriores. O Hotpotatoes permite exportar seu conteúdo nos formatos página web, pacote zip, SCORM e para WebCT. Os exercícios construídos por esta ferramenta podem ser utilizados como parte de um tutorial promovendo a avaliação formativa e, se exportados no formato SCORM e inclusos no LMS Moodle, poderão ser utilizados também como avaliação somativa.

Conforme Santanchè et al (2008), a ferramenta CourseLab é gratuita e utiliza a metáfora de slides para sistematizar o conteúdo produzido. Dispõe de uma rica galeria de recursos prontos, bem como suporte à construção de animações. Tal como acontece no eXe, além de dispor de múltiplas formas de criação de conteúdo, o CourseLab também pode ser

\footnotetext{
${ }^{6}$ Mais informações disponíveis no site http://www.reload.ac.uk/index.html
} 


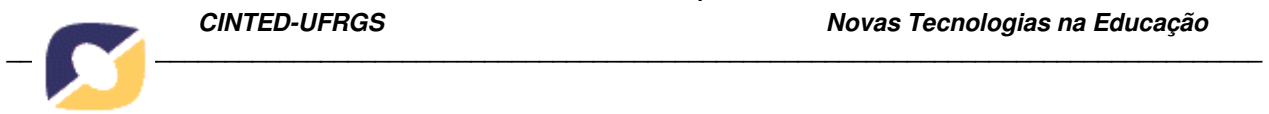

usado para a criação de atividades e questionários, que enviam os resultados do desempenho do estudante para um Sistema Gerenciador de Curso através da interface SCORM/AICC.

Thesis é uma ferramenta comercial da empresa HunterStone conhecida pela rápida conversão de conteúdos criados pelas ferramentas do pacote Office para o formato SCORM. (HUNTERSTONE, 2007)

Thesis permite que conteúdos produzidos nas ferramentas do pacote Office da Microsoft sejam "scormizados" através de uma opção que é disponibilizada na interface do próprio Office, semelhante a opção salvar como.

\section{Estudo de Caso}

Atualmente, no ensino de graduação, as aulas predominam no modo presencial. Quando se quer introduzir modificações usando ferramentas que implementam padrões e comportamentos dentro dos sistemas de gestão, há uma certa relutância em aceitar. Na disciplina de Parasitologia e Micologia do curso de Medicina da Universidade Federal de Ciências da Saúde de Porto Alegre (UFCSPA), os alunos da primeira série do curso, sempre tiveram suas aulas no modo presencial. A partir do ano de 2009, a disciplina foi inserida no sistema Moodle da Instituição e parte dela teve atividades na modalidade a distância com o uso das ferramentas deste ambiente. Foram preparados casos clínicos focados no "aprendizado baseado em problema" (Problem basead Learning - PBL), onde a resposta deve ser obtida pela solução do caso a ele apresentado. Os alunos recebem o caso clínico e respondem antes do professor apresentar os conteúdos que envolvem a solução do problema.

Numa informação prévia, foi solicitado de modo informal e escrito sem identificação, para que os alunos respondessem se esta nova estratégia de ensino teria ajudado na aprendizagem dos conteúdos da disciplina. Por unanimidade, todos consideraram ótima esta estratégia considerando importante que ela continue sendo usada nos próximos anos.

Para ilustrar esta pesquisa foi concebido um estudo de caso construído através da ferramenta eXe e incorporado na referida disciplina no ambiente Moodle. Os alunos deveriam postar suas respostas na atividade determinada através das instruções do estudo de caso. Como resultado desse processo pode se obter uma avaliação do aprendizado pelos alunos envolvidos.

O estudo de caso publicado no LMS Moodle foi produzido através da ferramenta de autoria eXe. Como foi dito na seção 3.1 uma unidade de aprendizagem é composta por vários iDevices, que são adicionados em páginas na sequência em que o professor desejar. Para isto, o eXe oferece controles de navegação permitindo que as páginas possam ser realocadas em qualquer posição e ainda em qualquer nível, como mostra a figura 3.

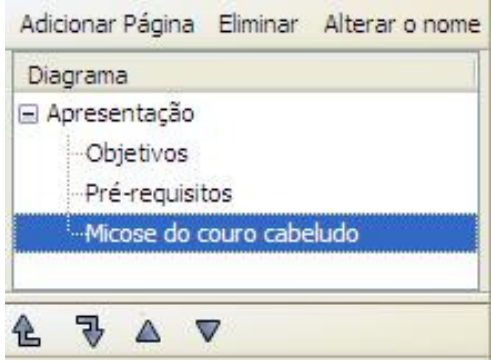

Figura 3 - Painel da Estrutura da Unidade

Para a construção do OA foram escolhidos os iDevices Texto Livre, Objetivos, Prérequisitos, Estudo de caso e Galeria de imagens, com o intuito de atender aos objetivos pedagógicos da atividade descritos no início desta seção. A figura 4 mostra a tela de edição do iDevice Texto Livre referente a apresentação do OA. 

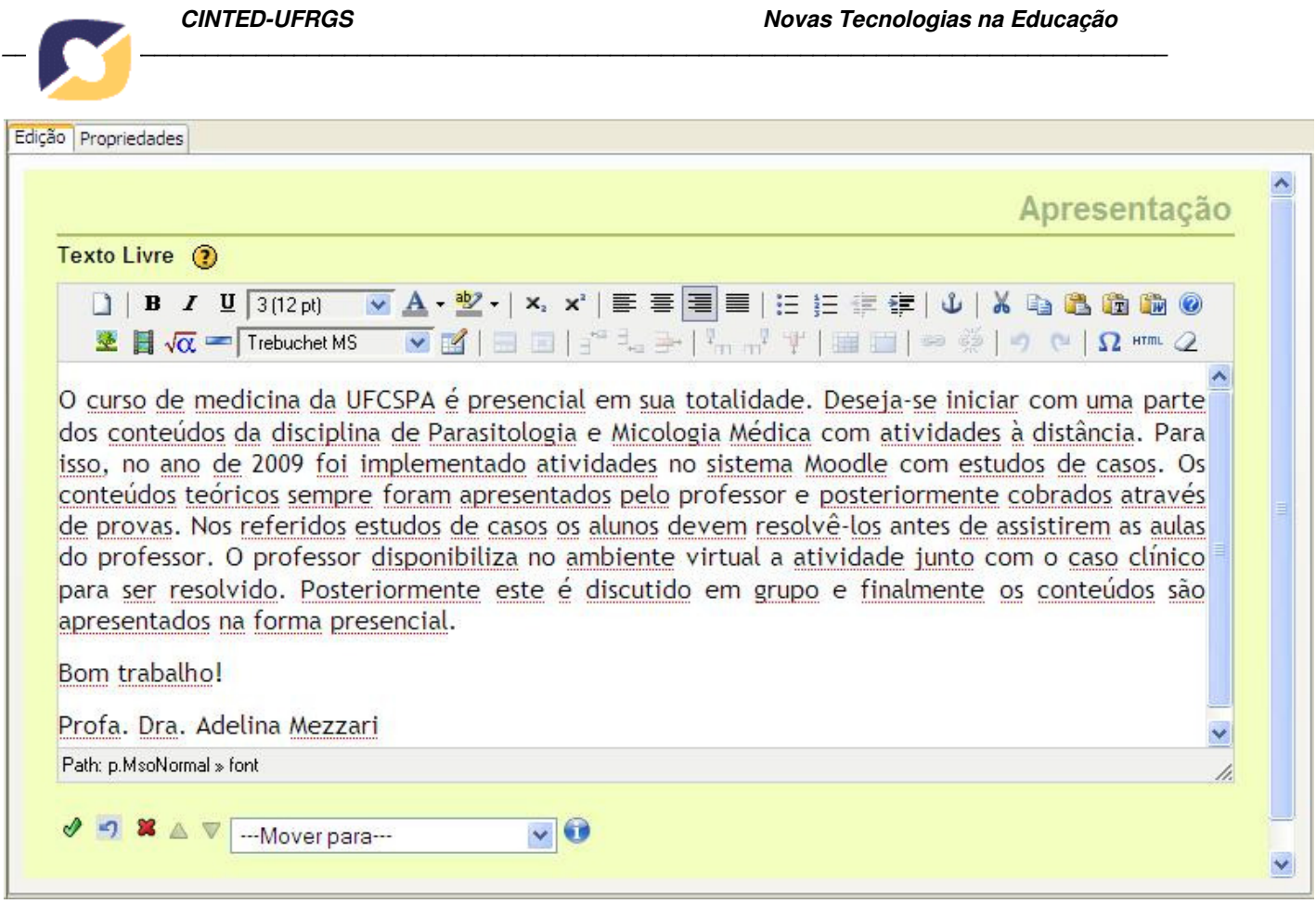

Figura 4 - Janela de edição do iDevice Texto Livre

Após a seleção dos iDevices e preenchimentos dos campos de cada um deles, o OA foi salvo como projeto para que posteriormente seja possível alterá-lo, caso necessário. Após salvo como projeto, o $\mathrm{OA}$ foi exportado em três formatos distintos possibilitando uma comparação entre eles e a escolha do formato adequado para publicação no Moodle. Os formatos exportados foram: SCORM 1.2, IMS Módulo de Conteúdo, Sítio Web - Pasta de Conteúdo.

\subsection{Ambientes que aceitam OAs empacotados segundo os padrões analisados}

Com base no estudo de caso construído na ferramenta eXe, foram realizados testes de importação no LMS Moodle, a partir de três formatos exportados pela ferramenta e aceitos pelo ambiente. Todos os testes foram bem sucedidos, desta forma a ferramenta eXe e o LMS Moodle são compatíveis, ou seja, o ambiente está adequado a receber pacotes nos formatos SCORM e IMS e ainda como pacote zip com conteúdo no formato sítio web, exportados pela ferramenta eXe.

Para importar no Moodle um pacote de conteúdo no padrão IMS utiliza-se o módulo MrCute 2, um módulo adicional do Moodle, que pode ser considerado um CMS (Content Management System) pela sua característica de repositório de objetos de aprendizagem, que permite importar pacotes no formato IMS e estes ficando disponíveis para todos os cursos do Moodle mantidos por uma mesma instalação/servidor.

O armazenamento do pacote (arquivo zipado) carregado é feito em uma parte da estrutura de arquivos do Moodle, externa a qualquer curso individual e pode ser configurado para ser compartilhado ou não. Isto permite que um educador cadastrado naquele servidor Moodle utilize a função de busca (usando palavras chave, categorias) para localizar conteúdos apropriados e incorporá-los a qualquer curso. Além disso, o módulo permite a criação de pacotes IMS a partir de conteúdos já criados. (TAROUCO et al, 2009)

A incorporação do OA ao LMS Moodle depende do formato que o conteúdo foi exportado. Para incorporar o conteúdo no formato IMS utiliza-se o módulo MrCute 2 disponível através das opções Acrescentar recurso Find ready made materials e Upload learning materials. Na incorporação do conteúdo "scormizado" a opção indicada é Acrescentar atividade SCORM, já para incorporar um conteúdo no formato Sítio Web - pasta de conteúdo a opção indicada é Acrescentar recurso - Link a um arquivo ou site.

A diferença de visualização entre os conteúdos de diferentes formatos é mais evidente entre os formatos de sítio web e os demais, que são quase idênticos. A figura 5 apresenta o OA 
no formato SCORM, onde pode observar após o uso do OA a rastreabilidade da navegação do usuário pelo conteúdo, indicadas a partir de um ticket na cor verde ao lado de cada página do conteúdo. O objeto de aprendizagem, por meio do formato SCORM, permite que a navegação seja realizada através do menu disponível a esquerda ou através dos botões de navegação (Anterior | Próximo) disponíveis dentro do conteúdo da página.

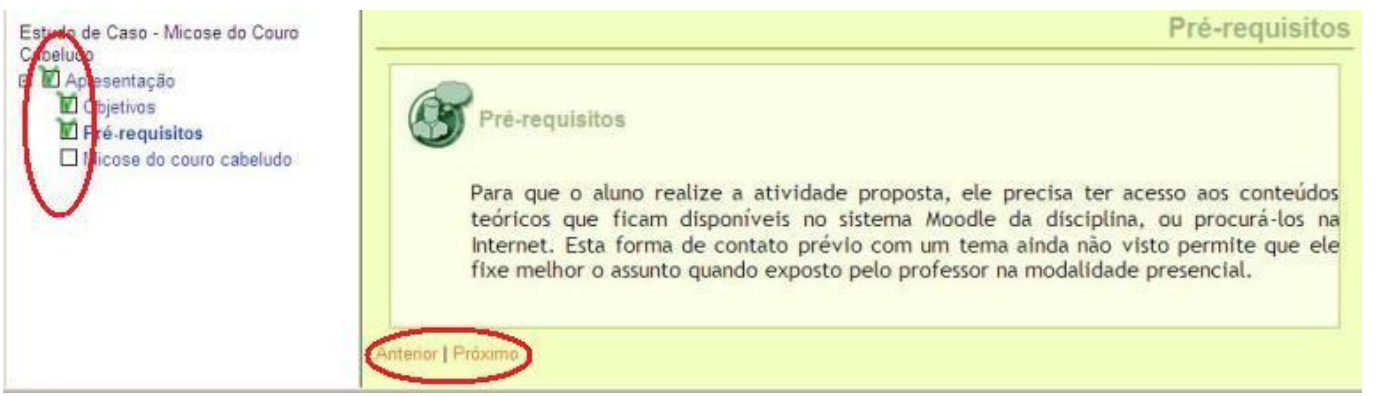

Figura 5 - OA no formato Scorm 1.2

A figura 6 apresenta o mesmo OA no formato IMS importado no Moodle através do módulo MrCute 2. Percebe-se que a diferença entre este formato e o anterior está na limitação de não rastrear a navegabilidade do aluno pelo $O A$ e não oferecer a possibilidade em navegar através de botões dentro do próprio conteúdo, assim o aluno para evoluir na navegação deve sempre dirigir-se ao menu à esquerda do conteúdo.

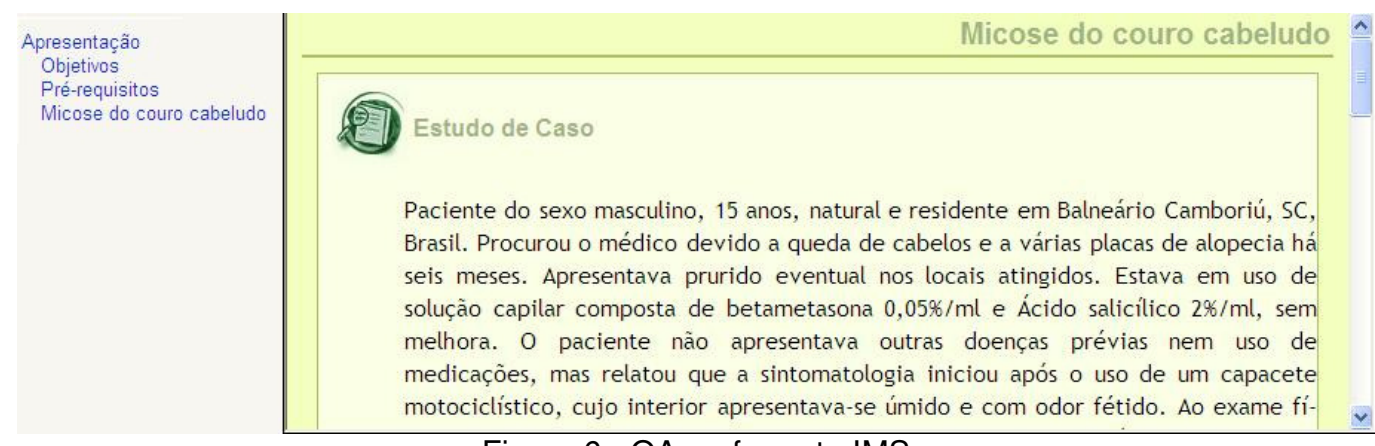

Figura 6 - OA no formato IMS

No formato de página Web importado no Moodle são apresentados os botões de navegação junto ao conteúdo permitindo ao aluno duas formas de navegação, mas com os limitadores de não rastrear a navegação e não registrar a avaliação no LMS.

No que tange a avaliação, a diferença está que no formato SCORM a navegação realizada por cada aluno fica registrada no ambiente possibilitando que o aluno, em uma nova visita ao OA, verifique o seu rastro, ou seja, as páginas que ele já percorreu. Além disso, quando utilizamos um iDevice do tipo SCORM Quiz (somativo) na construção de uma atividade do OA na ferramenta eXe, o resultado da interação de cada aluno com tal atividade fica registrado no Moodle na forma de nota, considerando o percentual de acertos.

Além dos testes de importação realizados com a ferramenta eXe, foram testadas também as ferramentas Thesis e Módulo Livro do Moodle. A ferramenta Thesis permite salvar os arquivos produzidos pelo pacote Office no formato SCORM. E o recurso livro do próprio Moodle permite empacotar o seu conteúdo no padrão IMS. Ambos os conteúdos testados foram incorporados ao Moodle com sucesso, mas com a limitação de não armazenar a rastreabilidade da navegação do usuário e o registro de avaliação.

\section{Conclusões}

Os OAs disponibilizados via Internet através de ambientes virtuais de aprendizagem como o Moodle, oportunizam a comunicação e a interação facilitando o processo de ensinoaprendizagem. É importante que estes OAs sejam projetados e construídos conforme o 
contexto desejado e posteriormente empacotados a partir de um dos padrões existentes para que possam ser gerenciados quanto ao seu acesso pelos alunos e reutilizados em outras situações de aprendizagem.

Foram estudadas algumas ferramentas de autoria de materiais educacionais digitais e foi constatado que as mesmas permitem o encapsulamento de objetos em pelo menos um dos padrões, SCORM ou IMS, através de opções de exportação disponíveis nas próprias ferramentas.

O LMS ao disponibilizar os pacotes "scormizados" registra a avaliação formativa e somativa do aprendizado. O aluno é avaliado pela sua interação com o objeto disponibilizado no ambiente virtual. O resultado desta interação fica registrado na forma de nota quando a atividade gerada na ferramenta de autoria utilizada é do tipo SCORM. Já os OAs empacotados no padrão IMS é possível que o aluno faça apenas a avaliação formativa, verificando as suas necessidades de acordo com a interação com o objeto.

Como trabalhos futuros considera-se que são necessários mais experimentos relacionados ao uso, projeto, construção e gestão de objetos de aprendizagem. Existe a necessidade de um padrão para integração entre os LMSs e os repositórios de aprendizagem existentes, viabilizando desta forma a reutilização de OAs.

\section{Referências}

ADVANCED DISTRIBUTED LEARNING (ADL). Estados Unidos: [s,d.]. Disponível em: <http://www.adlnet.org> Acesso em: 15 jul. 2004.

ARIADNE. (2000). Alliance of remote instructional authoring and distribution networks for Europe website [On-line]. Disponível em: <http://ariadne.unil.ch/> Acesso em: 15 jul. 2004.

DUTRA, Renato Luís de Souza; TAROUCO, Liane Margarida R. Recursos Educacionais Abertos. RENOTE. Revista Novas Tecnologias na Educação, v. 5, p. 1-8, 2007.

DUTRA, Renato Luís de Souza. Encapsulamento e utilização de objetos de aprendizagem abertos SCORM para ensejar a avaliação formativa. 2008. 173 f. Tese (Doutorado) - Curso de Informática Na Educação, Departamento de Programa de Pós-graduação em Informática $\mathrm{Na}$ Educação, Universidade Federal do Rio Grande do Sul, Porto Alegre, 2008.

HARMAN, Keith; KOOHANG, Alex. ed. (2007a) Learning objects: Applications, Implications and Future Directions. Santa Rosa, Califórnia: Informing Science Press, 2007.

HARMAN, Keith; KOOHANG, Alex. ed. (2007b) Learning objects: standards, metadata, repositories, and LCMS. Santa Rosa, Califórnia: Informing Science Press, 2007.

HUNTERSTONE. Leaders in SCORM eLearning and Microsoft Related Services. S. local: 2007. Disponível em: <http://www.hunterstone.com/> Acesso em: 19/10/2009.

IMS. (2000). Instructional management systems project website [On-line]. Disponível em: $<$ http://imsproject.org/imMembers.html>

KOOHANG, Alex; HARMAN, Keith. ed. (2007a). Learning Objects: Theory, Praxis, Issues and Trends. Santa Rosa, Califórnia: Informing Science Press, 2007.

KOOHANG, Alex; HARMAN, Keith. ed. (2007b) Learning Objects and Instructional Design. Santa Rosa, Califórnia: Informing Science Press, 2007.

LTSC. (2000a). Learning technology standards committee website [On-line]. Disponível em: < http://ltsc.ieee.org/> Acesso em: 15 jul. 2004.

LTSC. (2000b). IEEE standards board: Project authorization request (PAR) form [On-line]. Disponível em: <http://ltsc.ieee.org/par-lo.htm> Acesso em: 15 jul. 2004.

Presentations ICOPER project. Disponível em: <http://www.icoper.org/outreach/presentations>. Acesso em: 12 out. 2009.

SANTANCHÈ, André; LAGO, Andrea; DOURADO, Patrícia; FILHO, Pedro Ferreira. Ferramentas e Ambientes para Objetos de Aprendizagem. Minicurso ministrado no SBIE 2008, 12 a 14 de Novembro de 2008, Fortaleza, CE.

V. $7 \mathrm{~N}^{\mathrm{o}}$ 3, dezembro, 2009 
TAROUCO, Liane M. R.; KONRATH, Mary Lúcia Pedroso; DUTRA, Renato Luís de Souza. IMS Learning Design, evoluindo de Objetos de Aprendizagem para Atividades de Aprendizagem. RENOTE. Revista Novas Tecnologias na Educação, Porto Alegre, v. 3, n. 1, p. 1-8, 2005.

TAROUCO, Liane M. R.; DUTRA, Renato Luis de Souza. Padrões e interoperabilidade. In: Carmem Lucia Prata, Anna Christina Aun de Azevedo Nascimento. (Org.). Objetos de Aprendizagem - Uma proposta de recurso pedagógico. Brasília - DF: MEC/SEED, 2007.

TAROUCO, Liane M. R.; DUTRA, Renato Luís de Souza; ÁVILA, Bárbara Gorziza; GRANDO, Anita Raquel da Silva. SCORM e portabilidade: motivação e possibilidades. RENOTE. Revista Novas Tecnologias na Educação, v. 5, p. 1-10, 2007.

TAROUCO, Liane Margarida R.; SCHMITT, Marcelo Augusto Rauh; RODRIGUES, Alessandra Pereira; VICCARI, Rosa Maria. Gestão colaborativa de conteúdo educacional. RENOTE: Revista de Novas Tecnologias na Educação, Porto Alegre, v. 7, n. 1, p.1-13, 01 jul. 2009. Semestral. Disponível em: <http://www.cinted.ufrgs.br/renote/jul2009/artigos/5b_liane.pdf>. Acesso em: 01 jul. 2009.

WILLEY, David A. (2002) Connecting learning objects to instructional design theory: A definition, a metaphor, and a taxionomy. Disponível em: <http://reusability.org/read/chpters/wiley.doc>. Acesso em: 07 out. 2009. 